\title{
INFLUENCE OF MEVALONIC ACID AND LINALOOL ON LIMONENE ACCUMULATION IN CALLUS TISSUES OF CITRUS GRANDIS OSBECK
}

\author{
NIK NORULAINI NIK AB RAHMAN', ZARINA ZAKARiA ${ }^{2}$.AND MOHD OMAR ADD KADIR ${ }^{\mathrm{S}}$ \\ 'School fur Distance Learning Education, Universiti Sains Malaysia, \\ 11800 USM. Penang, Malaysia. ${ }^{2}$ Faculty of Applied Sciences, \\ Universiti Teknologi MARA, Arau Campus, \\ 02600 Arau, Perils, Malaysia. \\ ${ }^{3}$ School of Industrial Technology, Universiti Sains Malaysia, \\ 11800 USM, Penang, Malaysia.
}

\begin{abstract}
Effect on callus growth was studied for Citrus grandis cultured with feeding of exogenous mevalonic acid (MVA) at concentrations of 0.04, 0.08, 0.38, 0.77, 1.15 and $1.54 \mathrm{mM}$. Similar effect with linalool ranging from 10 to 200 $\mathrm{nl}$ was studied under various incubation periods. The growth was proportional to the concentrations of precursors used meaning that higher precursors concentrations influenced more growth on $C$. grandis callus culture. Mevalonic acid and linalool showed quite similar precursor feeding effects on limonene accumulation of $C$. grandis callus cultures. It was revealed that limonene production was triggered with the introduction of MVA and linalool even at low concentration. Limonene accumulation was detected as early as week four and continued to increase at about 0.0030 and $0.0032 \mathrm{mg} / \mathrm{g}$ with MVA and linalool, respectively, after the seventh week incubation. In comparison to the unfed cultures, no limonene was detected from the callus up to eight weeks in incubation.
\end{abstract}

Keywords : C/rrusgrarafo/Limonene/Linalool/Mevalonic acid

Abbreviations: 2,4-D: 2,4—Dichlorophenoxyacetic acid, ABA: Abscisic acid, MVA : Mevalonic acid

\section{INTRODUCTION}

Bioconversion using an exogenous supply of biosynthetic precursor is believed to improve the accumulation of desired metabolites compound where the productivity is limited because of the lack of particular precursors. Naturally occurring as well as related synthetic compounds may be used as precursors. Many attempts have been made to produce valuable compounds by adding precursors to several culture species. Often the precursors undergo more than one bioconversion resulting in complex mixture of unknown products (Kawaguchi et al. 1988). Dorisse et al. (1988) had studied the bioconversion of papaverine in Glycyrrhiza gabra cell suspension and found the bioconversion rate into papaverinol (31.5\%), papa-veraldine (4\%) and demethylpapaverine was rather slow. In other case, hydro-

quinone was glucosylated into arbutin after addition to cell suspension cultures of

Datura innoxia (Suzuki et al. 1987) in production rate of 2.5 gl-1 after of incubation 
Feeding of $3 \mathrm{mM}$ L-tyrosine or phenylalanine into Lithospermum flavum cultures yielded a 2-3 fold increase in 5-methoxy podophyllotoxin accumulation

(Van Uden et al. 1990). The byconversion rates were low; 0.34 and $0.5 \mathrm{mg} \mathrm{g}$ d, respectively. It is clear that there are significant differences in bioconversion rates. Several reasons include that; the rate may be dependent on the solubility of precursors and/or the intracellularly present amounts of enzyme. Furthermore, the precursors can be metabolized by other reactions and even a specific transport system may be involved. Funk and Brodelius (1990) have used precursor feeding and addition of metabolic inhibitors techniques as a tool to evaluate the possibility that involved to the undetactable vanillin from the cultivated cells of Vanilla plan/folia. They found that cinnamic acid, but not ferulic acid, is a precursor of vanillic acid in these cultivated cells of $V$. plamfolia.

Extraction of Citrus grandis or pomelo peel contained almost exclusively limonene (92.6\%) and myrcena (1.9\%) suggests that pomelo may be utilized as source material for the production of limonene (Wong 1992). The precursors for the synthesis of monoterpenoids are obtained from acetate-mevalonate pathway, which supply to mevalonic acid. Mevalonic acid is the primary precursor of all the terpenoids biosynthesized by plant, including limonene. Orange juice vesicles (Citrus sp., Rutaceae) contain an enzyme system which is capable of phospho-rylating mevalonic acid and forming the final product, a monoterpene, linalool. Intact fruits and leaves convert linalooi to limonene and other cyclic monoterpenoids. Linalool is considered as an immediate precursor which is expected to readily converted to limonene under favourable conditions.

There are numerous reports of experiments on mevalonic acid as a precursor. Moreno et al. (1993) reported that feeding mevalonic acid did not increase alkaloid accumulation in cell suspension cultures of Catharanthus roseus. Mevalonic acid

added to cultures at concentration of $3.3 \mathrm{mM}$ was reported to saturate the 030 (steroid) biosynthetic pathway in Nicotiana tabaccum suspension cultures (Threfall \& Whitehead 1988). With the aim of shortening the period for limonene production and enhancing its accumulation, the effect of feeding primary and immediate precursors was investigated.

\section{MATERIALS AND METHODS}

\section{Culture conditions}

Young C. grandis fruits about five weeks after anthesis and 4-5 cm in diameter were obtained from established plantation. Sterilization technique was established by immersing the whole fruit into $20 \%$ Clorox ${ }^{\circledR}$ (5.2\% sodium hypochloride) for 2 hours prior to peeling and cutting. The fruits were rinsed with sterile distilled water three times and cut transversely into small segments. Callus was initiated on MS (Murashige \& Skoog 1962) basal medium supplemented with 30 $\mathrm{mg} / 1$ sucrose. $3.0 \mathrm{me} / 1$ 2.4-D $3.0 \mathrm{mg} / 1$ kinetin and $0.2 \mathrm{mg} / 1 \mathrm{ABA}$ and incubated in the dark 24 
Influence of mevalonic acid and linalool on limonene accumulation - Nik Norulaini Nik Ab Rahman et al.

hours/day at room temperature. Callus were formed after 2-6 weeks with a success rate of $80-90 \%$. Callus were maintained on the media and subcultured to the same fresh media every 4 weeks interval.

\section{Feeding of precursors}

Mevalonic acid lactone (MVA) and linalool (Analytical grades) were purchased from Fluka ( Japan). Aqueous solution of MVA was prepared by adding $50 \mathrm{ml}$ distilled water to $1 \mathrm{~g}$ of MVA powder. MVA was filter sterilized and aliquots were added to different MS media to get a range of final MVA concentrations of $0.04,0.08,0.38,0.77,1.15$ and $1.54 \mathrm{mM}$ in the media. Various volume of linalool ( 97\% purity), 10, 50, 100, 150 and $200 \mu \mathrm{j}$ were added to 1 liter MS media prior to autoclave. After 4 weeks, callus from maintenance media were cut into smaller pieces and transferred by spreading over the same MS medium with varying added precursors.

\section{Callus growth determination}

Callus fresh weight was taken as final weight started from the fourth week followed by fifth, sixth, seventh and eighth week of incubation period by direct measurement. To minimize the differences in growth, the initial weight of the callus prior transferring to the experimental MS media was ensured about equal according to each treatment. Callus was cleaned from attached media and dried using tissue paper before weighing using analytical balance (Sartorius, Germany). Several plates were sacrificed along the time course of the experiment, to measure the biomass accumulate.

\section{Limonene determination and analysis}

Extraction of limonene was carried out from four to eight weeks old callus. Fresh callus weighing $2.0-3.0 \mathrm{~g}$ were extracted with approximately $70 \mathrm{ml}$ methanol using soxhlet apparatus according to Morris et al. (1985). Identifications and quantification of limonene were made by comparing with standard purchased from Fluka using Gas Chromatography (Shimadzu GC 17A) equipped with FID detector. Column used was BP-20 (0.25 (im, 30m X $0.25 \mathrm{~mm})$ from $\mathrm{SGE}$. The temperature was programmed at $60 \mathrm{C}, 4 \mathrm{~min}, 140 \mathrm{C}, 8 \mathrm{~min}$. Injector and detector temperature was $250 \mathrm{C}$ respectively. The experiments were performed in triplicates and data were subjected to analysis of variance (ANOVA). 


\section{RESULTS}

\section{Effect of various concentrations of MVA feeding and incubation period on callus growth rate}

Figure 1 shows the effect of various concentrations of MVA feeding on callus fresh weight. Comparisons of callus fresh weights, taken as an indicator of cell proliferation were determined within the eighth weeks period for all MVA feeding tested. Addition of exogenous MVA enhanced the callus growth even at low level except for $0.04 \mathrm{mM}$ compared to the control. However, as the concentration of MVA feeding increased, the callus fresh weight increased concomitantly as pointed out in the figure. Among the concentrations used, there was no significant difference ( $\mathrm{P}>0.05$ ) between 0.38 and $0.77 \mathrm{mM}$ and between 1.15 and $1.54 \mathrm{mM}$ (Table 1). Highest callus fresh weight was obtained with the feeding of 1.15 and $1.54 \mathrm{mM}$ of MVA where increase in fresh weight was about two times higher compared to control callus.

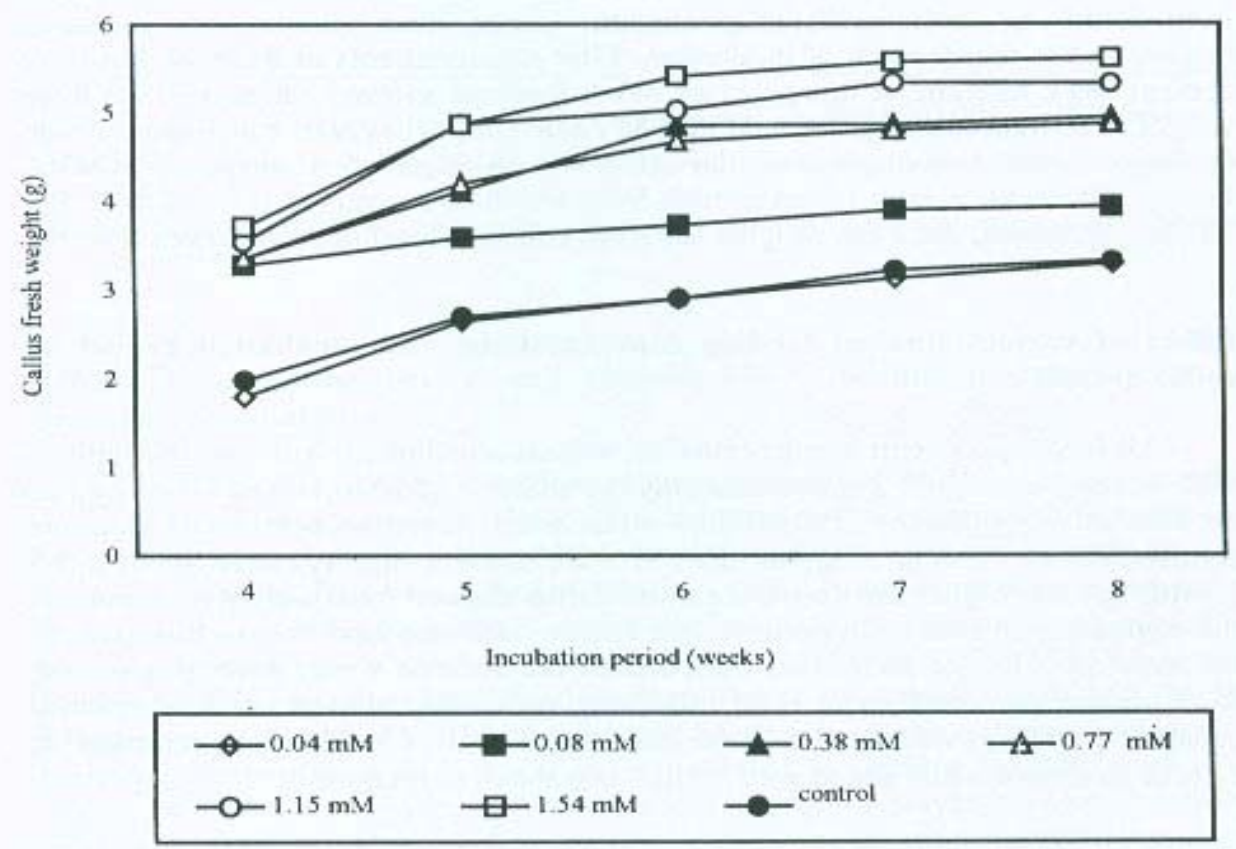

Figure 1 Time course (4-8 weeks) of callus growth in the presence of various concentrations of MVA feeding. 
Influence of mevalonic acid and linalool on limonene accumulation - Nik Norulaini Nik Ab Rahman et al.

Table 1: Effects of mevalonic acid concentrations on callus growth and limonene accumulation of Citrus grandis after culture for 7 weeks.

\begin{tabular}{|c|c|c|}
\hline \multicolumn{2}{|c|}{ MVA(mM) } & Fresh weight (g) \\
\hline 0.04 & $3.127 a$ & $0.0015 c$ \\
\hline 0.08 & $3.900 \mathrm{C}$ & $0.003 \mathrm{le}$ \\
\hline 0.38 & $4.868 d$ & ๑.0030e \\
\hline 0.77 & $4.807 d$ & $0.0020 d$ \\
\hline 1.15 & $5.335 \mathrm{e}$ & $0.00 \mathrm{ISC}$ \\
\hline 1.54 & $5.574 \mathrm{e}$ & $0.0013 \mathrm{C}$ \\
\hline control & $3.218 b$ & $\odot$ \\
\hline
\end{tabular}

Different alphabet for each data indicates a significant difference at a =

0.05 according to Tukey HSD test.

The figure also reveals that MVA feeding exerts positive effect on callus growth throughout the eight-week culture duration. The most active growth occurred from fourth to sixth week in incubation. Callus fresh weight was measured beginning the fourth week of incubation. First measurements of fresh weight were done at week four and at this point all MVA feedings showed a higher callus fresh weight than control except for 0.04 mM MVA feeding. However, subsequently each feeding concentration displays similar callus growth. Higher concentrations of MVA feeding illustrates greater callus growth from fourth to seventh week in culture. For all corcentrations, the fresh weights achieved constant level after the seventh week.

\section{Effect of various linalool feeding concentrations and incubation period on callus growth rate}

All feeding concentrations of linalool enhanced callus growth rate, though to a different degree. Figure 2 shows that only the addition of 10 to $100 \mathrm{jil} / 1$ had resulted on distinctively different $(\mathrm{P}<0.005)$ in callus fresh weight proportional to linalool concentrations. Feeding of higher than 100 (j.1/1 showed slightly reduction in callus growth but still higher than control callus. Callus showed fresh weight increment at the beginning of incubation period at the fourth week and increased in fresh weight occurred after longer incubation period until the seventh week. After this period, fresh weight remained about level indicating callus had slowed down or reached constant growth. It was about twofold increment in callus fresh weight compared to control treatment when added with 50 (il/l and above of linalool. 


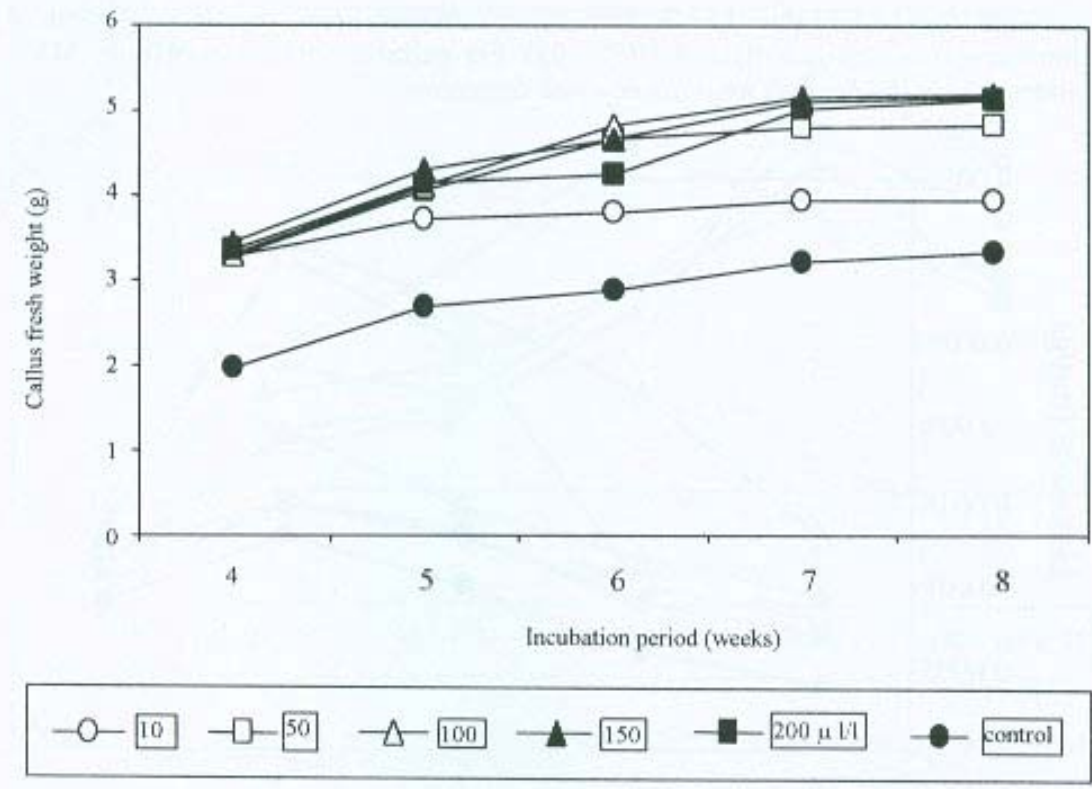

Hifure 2. I ime course (4-8 wecks) of callus growth in the prescnce of varwous concentrations of linaloof fexding

\section{Effect of incubation period and various MVA feeding concentrations on limonene accumulation}

The combined effect of various concentrations of MVA feeding and the incubation period is shown in Figure 3. Compared to cultures with no exogenous MVA added, other cultures with MVA feeding showed limonene as early as four weeks in culture. This accumulation continues up to the seventh week, whereby limonene accumulation displays a sudden decline. This phenomenon was exhibited in all MVA concentrations used. However, cultures fed with $0.08 \mathrm{mM}$ MVA shows a more active limonene accumulation from fourth to seventh week and this is followed by $0.38 \mathrm{mM}$ MVA which after the fifth week, shows a drastic increase in limonene accumulation to match that accumulated in cultures fed with $0.08 \mathrm{mM}$ MVA. Other concentrations of MVA feeding profile displayed an increase from fourth to seventh week and a decline thereafter in much lower limonene accumulation.

Greatest effect of MVA concentrations was observed on limonene accumulation using 0.08 and $0.38 \mathrm{mM}$, whereby there is no significant difference 
Influence of mevalonic acid and linalool on limonene accumulation - Nik Norulaini Nik Ab Rahman et al.

between the two (Table 1). Outside these concentrations, the accumulation of limonene was significantly lower $(\mathrm{P}>0.05)$. For cultures with no exogenous MVA added that is the control, no limonene was detectable.

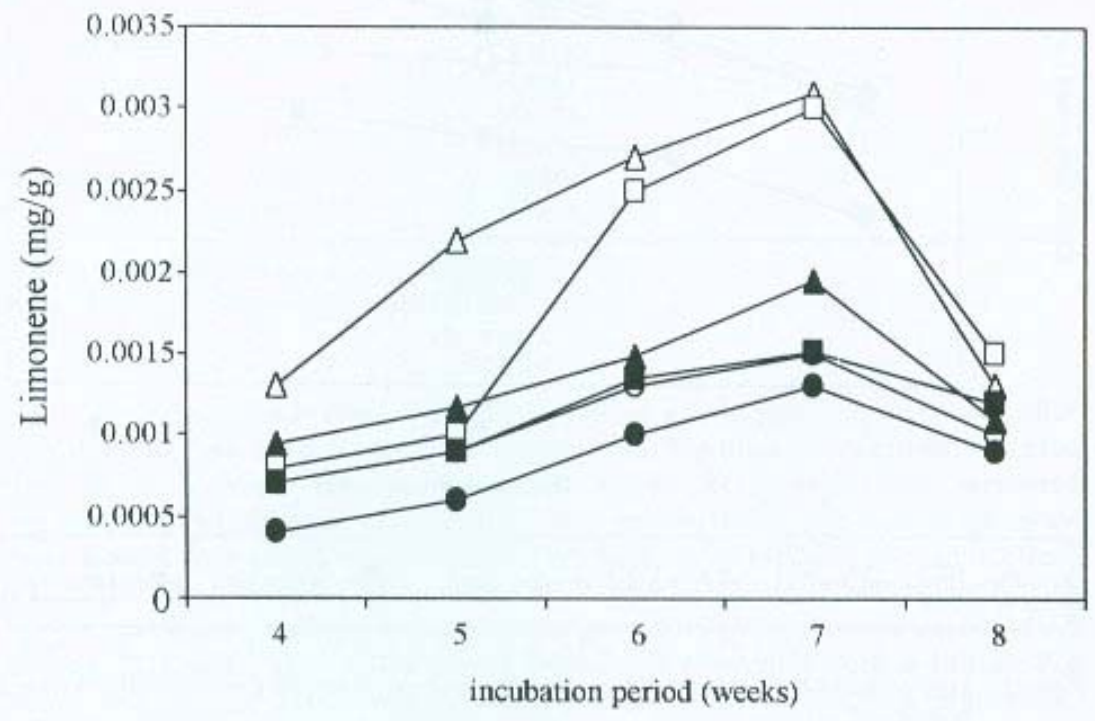

$\begin{array}{llll}-0-0.04 \mathrm{mM} & \triangle 0.08 \mathrm{mM} & -\square-0.38 \mathrm{mM} & -0.77 \mathrm{mM} \\ -1.15 \mathrm{mM} & -1.54 \mathrm{mM}\end{array}$

Figure 3. Time course (4-8 woeks) of limonene accumulation in the presence of various concentrations of MVA. Nu limonene was detected in control.

\section{Effect of various linalool feeding concentrations and incubation period on limonene accumulation}

Results from Figure 4 shows that limonene accumulation was detected from feeding of linalool at all concentrations studied. There was no particular pattern on limonene accumulation with various linalool feeding concentrations. Limonene content seems to accumulate at a significant amount when 10 and $50 \mu \mathrm{l} / 1$ of linalool was added. This amount slightly decreased with feeding of $100 \mu \mathrm{l} / 1$ of linalool and increased instantly with $150 \mu .1 / 1$ before dropped again at concentration of $200 \mu .1 / 1$ of linalool. 


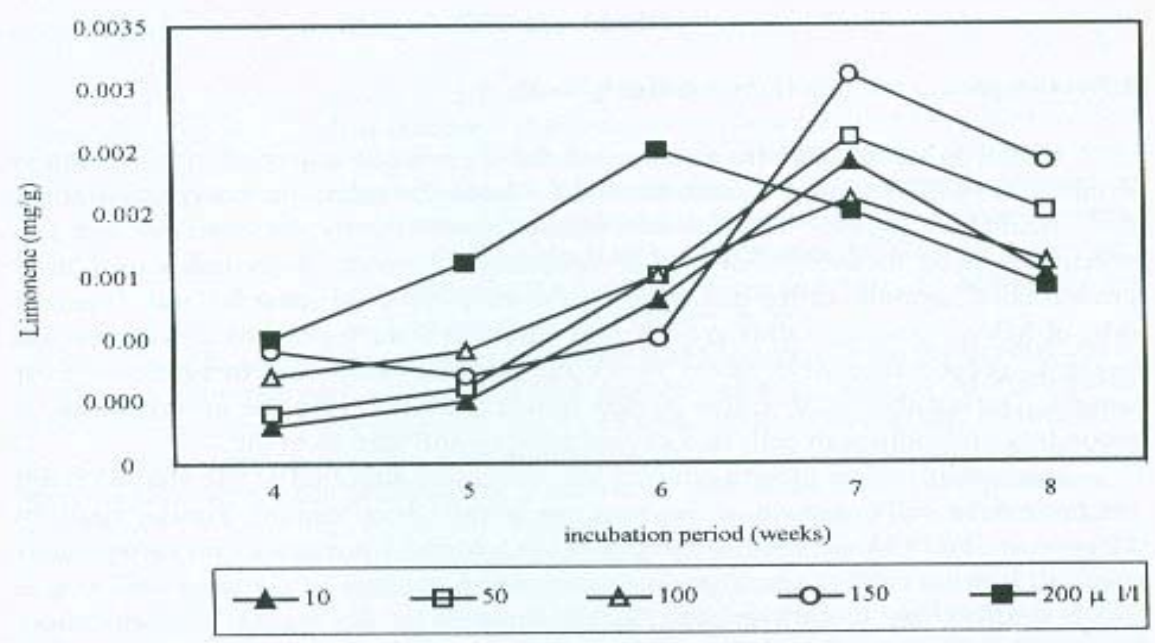

Figure 4 Time cours: (4-8 weeks) of Imanene accumulation th varwous cuncintratons of iceding linainol. No limonene was detected in contrul.

The figure also demonstrated that limonene accumulation was highest at seventh week of incubation period. However, limonene accumulation with $200 \mu \mathrm{l} / 1$ exogenous linalool addition gave maximum peak at week six. Limonene value dropped after the seventh week of incubation regardless of linalool feeding concentrations. Most rapid increase of limonene accumulation occurred between six to seven weeks of incubation and the feeding of $150 \mu \mathrm{l} / 1$ of linalool giving the highest accumulation (Table 2).

Table 2. Effects of linalool concentration on callus growth and limonene accumulation of Citrus grandis after culture for 7 weeks.

\begin{tabular}{ccc}
\hline Linaiool $(\mu \mathrm{l} / \mathrm{l})$ & Fresh weight $(\mathrm{g})$ & Limonene $(\mathrm{mg} / \mathrm{g})$ \\
\hline 10 & $3.933 \mathrm{~b}$ & $0.0024 \mathrm{~b}$ \\
50 & $4.182 \mathrm{c}$ & $0.0026 \mathrm{~b}$ \\
100 & $5.171 \mathrm{~d}$ & $0.0021 \mathrm{a}$ \\
150 & $5.124 \mathrm{~cd}$ & $0.0031 \mathrm{c}$ \\
200 & $5.033 \mathrm{~d}$ & $0.0020 \mathrm{a}$ \\
control & $3.218 \mathrm{a}$ & 0 \\
\hline
\end{tabular}

Different alphabet for each data indicates a significant

difference at $\alpha=0.05$ according to Tukey HSD test. 
Influence of mevalonic acid and Imalool on limonene accumulation - Nik Norulaini Nik Ab Rahman et al.

\section{DISCUSSION}

\section{Effect of precursor feeding on callus growth}

Effect on callus growth was studied for $C$. grandis cultured with feeding of exogenous MVA ( Figure 1) and linalool ( Figure 2) under various concentrations and incubation period. The growth was proportional to the concentrations of precursors used meaning that higher precursors concentrations influenced more growth on C. grandis callus culture except at very low concentration, which is $0.04 \mathrm{mM}$ of MVA. Obvious callus growth was observed from week five to week six and began to reduce after week seven where the increase was about threefold by fresh weight. During this time, callus turned brown indicated that the accumulation of secondary metabolites in cells had caused toxicity and started to die.

Increase in callus growth showed that precursor supplied at the studied range did not inhibit callus growth in fact improve growth development. Similar result by Moreno et al. (1993) stated that feeding of the terpenoid precursors mevalonic acid, loganin, loganic acid or secologanin to suspension cultures of Catharanthus roseus did not affect the culture growth, as determined by dry weight concentrations measured at 72 and 120 hrs after feeding. These results demonstrated no toxic effect for all precursors added to the cultures.

Decreased in callus fresh weight after the seventh week could coincide with the initiation of secondary metabolites synthesize as cell growth approached stationary phase. Lindsey and Yeoman (1983) concluded that there is indirect relationship between cells growth and secondary metabolites accumulation. It is expected that secondary metabolites accumulation was stimulated only when cells slow down their rate of growth i.e. at the end of the logarithmic phase and the beginning of the stationary phase. Ketchum et al. (1999) showed that Taxus suspension cultures accumulated maximum levels of taxoid at the end of the growth phase. This conclusion could be related to the finding of this study. Interestingly, the rate of growth and highest fresh weight value attained for both precursors did not differ significantly $(\mathrm{P}>0.05)$. This concluded that both precursors have had same potential to serve as internal growth stimulator acting to shoot up various aspects of cell metabolism.

It was suggested that rapid growth at the early stage of callus was due to synergistic effect of feeding precursors and added growth factors. This phenomenon was demonstrated when 2,4-D was reduced in immobilized cultures of Catharanthus roseus cells that rised up the production of alkaloid (Tom et al 1991). Decrease in growth rate after week seven for fed cells was found a little greater than nonfed cells. Reasonable explanation for this phenomenon is that the accumulation of limonene and linalool themselves might have caused cells toxicity which led to cell fatality (Brown et al 1987; Charlwood \& Brown 1987). 


\section{Effect of precursors feeding on limonene accumulation}

Mevalonic acid lactone and linalool showed quite similar precursor feeding effects on $C$. grandis callus cultures. Illustrations in Figure 3 for MVA feeding and Figure 4 for linalool feeding revealed that limonene production was triggered with the introduction of MVA and linalool at low concentration. Limonene accumulation was detected as early as week four and continued to increase until the seventh week. The highest limonene accumulation was $0.0030 \mathrm{mg} / \mathrm{g}$ and $0.0032 \mathrm{mg} / \mathrm{g}$ obtained from cultures fed with $0.08 \mathrm{mM}$ of MVA for the former and $150 \mathrm{uM}$ of linalool for the later on the seventh week of incubation (Tables 1 \& 2).

The results proved that precursor feeding had triggered limonene production in C. grandis cultures earlier than non fed cultures as suggested by Moreno et al. (1993). They stated that exogenous supply of a biosynthetic precursor, secologanin, to the culture medium may improve metabolites, such as strictosidine and ajmalicine accumulation where the productivity is limited by lack of that particular precursor. It was showed when they added the terpenoid precursor during the growth phase, about 11-fold increase in strictosidine occurred $72 \mathrm{hrs}$ after feeding and decreased to 5 fold after 120 hrs. Similar pattern was observed from Imbault et al. (1996) on MVA feeding effects. They showed that the inhibition of endogenous MVA production by pravastatin treatment blocked the alkaloid biosynthesis; supplying such cells with exogenous MVA allowed accumulation of alkaloid, but did not result in incorporation of MVA. However, Moreno et al. (1993) did not find any significant increase in alkaloid level after mevalonic acid feeding, the basic precursor for all terpenoids, in Catharanthus roseus suspension cultures. For both treatments, it is concluded that limonene production was not dependent on amount of precursors added. It showed that only a small amount of MVA and linalool was needed as a stimulator for limonene production.

Other possibility that contributes to this finding was the facts that MVA must be catalytically phosphorylated by ATP and mevalonic kinase itself for the pathway to continue. Therefore, the lack of those co-factor and enzyme had restricted limonene production eventhough with the presence of high amount of precursor (Rohmer et al. 1996; Lichtenthaler et al. 1997). The strong role of enzymes and its incorporation with feeding precursor in study of biosynthesis was briefly described by Zenk (1991) in his review. Besides, for accumulation of a secondary compound to occur in cultured cells, it is clearly necessary that the system retains the ability both to synthesize and to store the product.

Trend in limonene accumulation shows that cell maturity has direct relation to secondary metabolite accumulation with both precursor feeding. It was obvious that feeding of both precursors accumulated highest peak of same amount of limonene at the same incubation period. Highest limonene accumulation at the seventh week suggested that cells had slowed down their division to prepare themselves for metabolite production. This result fulfilled the suggestion from Lindsey and Yeoman (1983) that rapidly growing cultures accumulate cells and not secondary products. A similar pattern of results was obtained from suspension culture of 
Influence of mevalonic acid and linalool on limonene accumulation - Nik Norulaini Nik Ab Rahman et al.

Podophyllum hexandrum on the production of podophyllotoxin (Pras 1992). Both situations demonstrated that the increase in metabolite compounds accumulation occurred only at the mature-death stage of the growth curve.

Consideration also has to take account for limonene itself an intermediate product and would be converted to other form of monoterpenoid. This situation is definitely uncontrollable because biosynthesis is a continuous process and when and how the bioconversion occurred need further and deeper study. Potential compounds synthesize from limonene are a-terpeniol, perillic acid and carveon. Further experiments on identifying the accumulation of those compounds in C. grandis cultures should be carried out to prove this statement. For future work on limonene improvement, it can be suggested that study on the regulation of enzymes involved in limonene biosynthesis could impose clearer picture of the biosynthetic pathway. The use of suspension cultures for fast limonene accumulation is among the future work that can be considered.

\section{CONCLUSION}

The period of limonene production in $C$. grandis callus culture was shortened and accumulation was enhanced by precursor feeding with MVA and linalool. Callus growth rate improved significantly in the presence of the precursors. This result also reconfirmed the presence of MVA and linalool in limonene pathway.

\section{ACKNOWLEDGEMENT}

The authors would like to thank Universiti Sains Malaysia for the research grant and the Ministry of Science, Technology \& Environment (MOSTE) for National Science Fellowship scheme.

\section{REFERENCES}

Brown, J.T., Hegarty, P.K., \& B.V. Charlwood. 1987. The toxicity of monoterpenoids to plant cell cultures. Plant Science 48,195-201.

Charlwood, B.V. \& J.T. Brown. 1987. Transport and storage of secondary metabolites in tissue cultured plant cells. Biochemistry Society Transactions. 61-63.

Dorisse, P., Gleye, \}., Loiseau, P., Puig, P., Edy, A.M. \& M. Henry. 1988. Papaverine biotransformation in plant cell suspension cultures. Jour. Natural Products 120,211-234.

Funk, C. \& P. Brodelius. 1990. Influence of growth regulators and an elicitor on phenylpropanoid metabolism in suspension cultures of Vanilla planifolia. Phytochemistry, 29(3), 845 - 848.

Imbault, N., Thiersault, M., Duperon, P., Benabdelmouna, A. \& P. Doireau. 1996. Pravastatin: A tool for investigating the availability of mevalonate metabolites for primary and secondary metabolism in Catharanthus roseus cell suspensions. Physiologica Plantarium 98, 803-809. 
Lichtenthaler, H.K., Rohmer, M. \& J. Schwender. 1997. Two independent biochemical pathways for isopentenyl diphosphate and isoprenoid biosynthesis in higher plants. Physiologica Plantarium 101, 643-652.

Lindsey, K. \& M.M. Yeoman. 1983. The relationship between growth rate, differentiation and alkaloid accumulation in cell cultures. Jour. Experimental Botany, 34,1055-1065.

Kawaguchi, K., Hirotani, M. \& T. Furuya. 1988. Biotransformation of digitoxigenis by cell suspension cultures ofStrophanthus amboensis. Phytochemistry. 27, 3475-3479.

Ketchum, R.E.B., Gibson, D.M., Croteau, R.B. \& M.L. Shuler. 1999. The kinetics of taxoid accumulation in cell suspension cultures of Taxus following elicitation with methyl jasmonate. Biotechnol. Bioeng. 62(1), 97105.

Moreno, P.R.H., Vander Heijden, R. \& R. Verpoorte. 1993. Effect of terpenoid precursor feeding and elicitation on formation of indole alkaloids in cell suspension cultures of Catharanthus roseus. Plant Cell Reports 12, 702-705.

Morris, P., Scragg, A. H., Smart, N. J. \& A. Stafford. 1985. Secondary product formation by cell suspension cultures. In: Dixon, R. A. (ed.), Plant Cell Culture. IRL Press, Washington.

Murashige, T. \& F. Skoog. 1962. A revised medium for rapid growth and bio assays with Tobacco tissue cultures, Physiologica Plantarium 15, 473.

Pras, N. 1992. Bioconversion of naturally occurring precursors and related synthetic compounds using plant cell cultures. Jour, of Biotechnology 26, 29-62.

Rohmer, M., Seeman, M., Horbach, S., Bringer-Meyer, S. \& H. Sahm. 1996. Glyceraldehyde 3-phosphate and pyruvate as precursors of isoprenic units in an alternative non-mevalonate pathway for terpenoid biosynthesis. Journal American Chemical Society 118, 2564-2566.

Suzuki, T., Yoshioka, T., Tabata, M. \& Y. Fuyita. 1987. Potential of Datura innoxia cell suspension cultures for glucosylating hydroquinone. Plant Cell Report 6, 275-278.

Threfall, D.R. \& I.M. Whitehead. 1988. Co-ordinated inhibition of squalene synthetase and induction of enzymes of sesquiterpenoid phytoalexin biosynthesis in cultures of Nicotiana tabaccum. Phytochemistry 27,2567 - 2580.

Tom, R., Jardin, B., Chavarie, C., Rho, D. \& J. Archambault. 1991. Effect of culture process on alkaloid production by Catharanthus roseus cells. Jour, of Biotechnology 21, 21-42.

Van Uden, W., Pras, N. \& Th. M. Malingre. 1990. On the improvement of the podophyllotoxin production by phenylpropanoid precursor feeding to cell cultures of Podophyllum hexandrum Royle. Plant Cell Tissue Organ Culture 23,217-224.

Wong, K.C. 1992. Flavour compounds from local aromatic plants, In: Proceedings of Asean Workshop On The Production Of Natural Flavours For Food, p. 66 - 67.

Zenk, M.H. 1991. Chasing the enzymes of secondary metabolism: plant cell cultures as pot of gold. Phytochemistry 30(12), 3861-3863. 